\title{
Hemobilia due to Spontaneous Rupture of Cystic Artery Pseudoaneurysm: A Rare Complication of Laparoscopic Cholecystectomy
}

\author{
Sameer Ashok Rege, Supreet Marathe, Omprakash Rohondia \\ Department of Surgery, Seth GS Medical College, Parel, Mumbai, Maharashtra, India.
}

\section{Corresponding Author: \\ Dr Sameer Rege \\ Email: drsamrege@gmail.com}

This is an Open Access article distributed under the terms of the Creative Commons Attribution License (creativecommons.org/ licenses/by/3.0).

Received : September 26, 2017

Accepted : May 5, 2020

Published : May 10,2020

\begin{abstract}
Background: Laparoscopic cholecystectomy is the commonest laparoscopic surgery performed. Various complications have been documented however isolated vascular complications are rare, require a high degree of suspicion and often pose a considerable diagnostic and therapeutic challenge. Case Report: We hereby report a lady with hemobilia due to spontaneous rupture of pseudo-aneurysm of cystic artery, successfully treated with endovascular emboilization. Conclusion: Cystic artery pseudo-aneurysm rupture can potentially lead to a fatal emergency due to rupture during surgery.
\end{abstract}

Keywords: Aneurysm, Cholecystectomy, Gallbladder Diseases, Hemobilia, Laparoscopy.

\section{Introduction}

Laparoscopic cholecystectomy (LC) is the gold standard treatment for cholelithiasis with the advantage of faster patient mobilization and hospital discharge. It is the most common laparoscopic procedure performed worldwide with known complications of bile leakage, hemorrhage, sub-hepatic abscess and retained gall stones [1]. Reports of development of isolated vascular complications post-LC are exceedingly rare which may unpredictably present either early or late in the post-operative course and thus require a high degree of suspicion and often pose a considerable diagnostic and therapeutic challenge. This case report describes cystic artery pseudo-aneurysm with rupture into the common bile duct presenting as hematemesis diagnosed on computerized tomography and treated successfully with embolization.

\section{Case Report}

A 40-year-old lady was referred to us in tertiary care centre with two episodes of hematemesis. She was operated for laparoscopic cholecystectomy 4 weeks ago at a peripheral centre for calculous cholecystitis. The surgery was uneventful, however she presented to the same hospital with acute pain in epigastrium and hematemesis about 25 days after the laparoscopic procedure. Gastroduodenoscopy (UGI scopy) performed was normal. Chest radiograph and serum amylase were within normal limits. Ultrasonography revealed minimally dilated common bile duct (CBD) of 6 $\mathrm{mm}$ in the hepatobiliary tree. Patient was subjected to endosonography, which revealed sludge in the CBD. A stent was placed in the CBD after a wide sphincterotomy. Patient was asymptomatic for 2 days following which she had an episode of massive hematemesis, followed by another. She was referred to our institution after about a month for further management.

Upon presentation to our centre, she had grade II pallor with stable vitals. Blood pressure was $110 / 60 \mathrm{mmHg}$ and heart rate was $98 / \mathrm{min}$. Epigastric and right upper quadrant tenderness was noted. Laboratory results were as follows: 
hemoglobin $7.5 \mathrm{~g} / \mathrm{dL}$, white cell count $9.2 \mathrm{~mol} / \mathrm{L}$, platelet count $256 \times 10^{9} / \mathrm{L}$, with mildly prolonged coagulation times, total bilirubin $2.8 \mathrm{mg} / \mathrm{dL}$, direct bilirubin $1.9 \mathrm{mg} / \mathrm{dL}$, alanine aminotransferase 160 IU/L (N: 13-40 IU/L), aspartate aminotransferase 232 IU/L (N: 10-42 IU/L), $\gamma$-glutamyltransferase $194 \mathrm{IU} / \mathrm{L}(\mathrm{N}:<50 \mathrm{IU} / \mathrm{L})$, and alkaline phosphatase 199 IU/L (125-240 IU/L). Serum amylase levels were normal. UGI scopy was performed which revealed blood drops along the stent that had been inserted previously at the peripheral centre. A subsequent computerized tomography (CT) revealed blush of contrast along the side of CBD near the clips [Fig.1].

Patient was subjected to hepatic angiography which revealed pseudo-aneurysm of the cystic artery with extravasation of the contrast into the CBD [Fig.2]. The right hepatic artery was embolized with coil and a post-procedure angiography showing no leak of contrast [Fig.3]. The patient tolerated the trans-arterial embolization (TAE) well with improvement in her hemoglobin levels over the following days and was discharged from hospital. The CBD stent was removed after 4 weeks and patient had no repeat bleeding episodes at 9 years of follow-up.

\section{Discussion}

Laparoscopic cholecystectomy is the gold standard for treatment of symptomatic gall stones [1]. It is reportedly associated with an incidence of $0.2-1 \%$ of biliary injuries and $0.25-0.5 \%$ of vascular injuries [2]. These may manifest intraoperative or post-operative. Isolated vascular injuries may have bleeding from cystic artery stump, portal vein, or hepatic artery. Contributory factors for intra-operative bleeds can be listed as aberrant vascular anatomy to gallbladder and liver, inadequate dissection, partial clipping of the artery and diathermy diffusion through the clips [3]. Delayed vascular injuries can either present as ischemic strictures or pseudo-aneurysm of the vessel due to the partial injury [2].

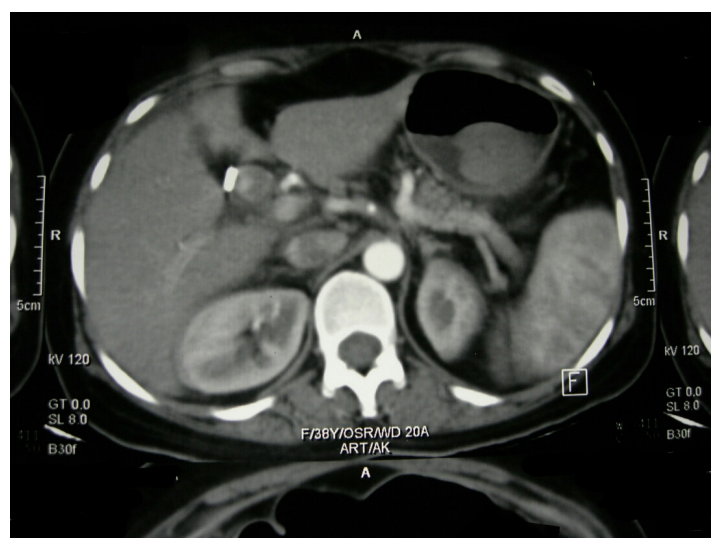

Fig.1: CECT scan abdomen showed active bluish adjacent to the surgical clips in porta.

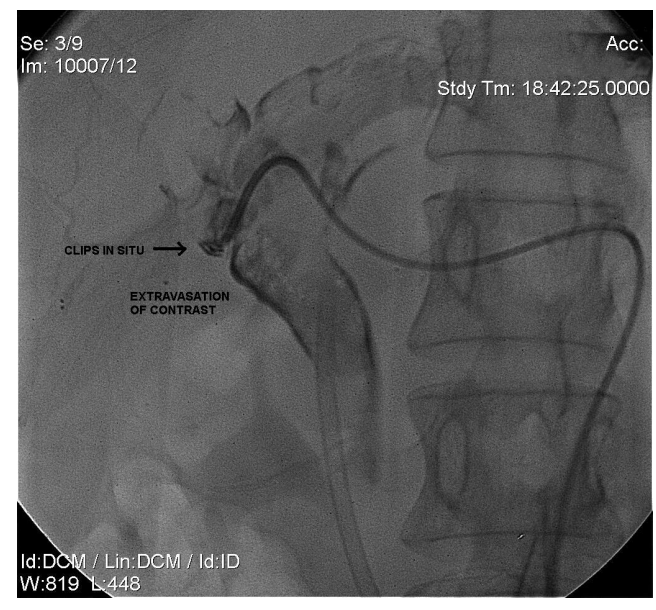

Fig.2: Hepatic angiography shows pseudo-aneurysm of the cystic artery with extravasation of the contrast into the CBD.

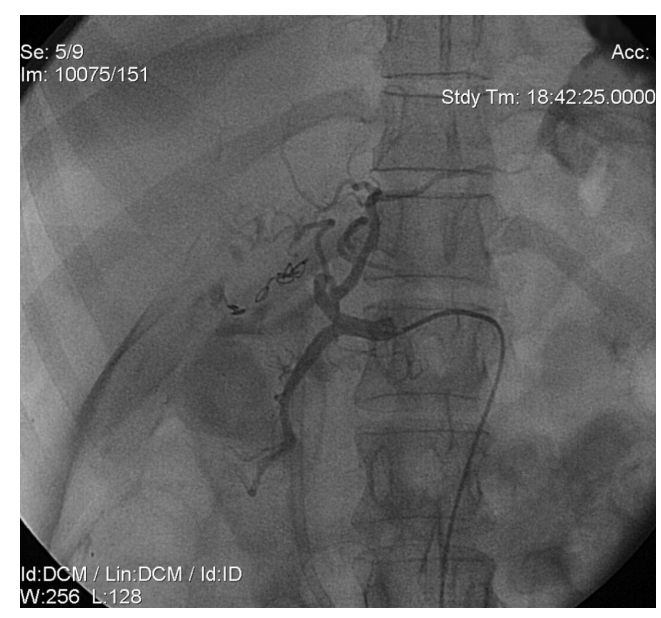

Fig.3: Successful coil embolization of the pseudo-aneurysm with no active extravasation. 
Pseudo-aneurysm of the cystic artery (PCA) is potentially fatal with impending rupture and hemobilia as complications. Hemobilia is bleeding into the biliary tree which occurs when there is a communication between a vessel and the intra-hepatic or extra-hepatic biliary system [2]. Only 20 cases of hemobilia secondary to PCA have been reported in the literature, only 9 cases following laparoscopic cholecystectomy as mentioned by Petrou et al. [2]. The mechanism is unclear but may involve direct mechanical vascular injury, thermal injury, and contributing factors include erosion due to clip intrusion, bile leakage, and infection [2]. The cytotoxic properties of high concentrations of intracellular or extracellular bile have been postulated to cause direct weakening of the suture line and to erode the vascular wall, leading to pseudo-aneurysm formation. Some authors have suggested that pseudo-aneurysms may result from post-operative bleeding from the cystic artery stump, with subsequent inflammation and erosion of the blood clot into the vessel wall [2]. In our case, partial clipping of the artery or vessel damage due to the clips seems to be the cause of PCA as the clips were seen immediately adjacent to the aneurysm. Hemobilia typically presents as Quincke's triad: colicky abdominal pain, jaundice and gastrointestinal hemorrhage [4]. Other presentations include hematemesis, malena, hemoperitoneum, altered liver enzyme profile (LFTs). An abdominal mass with bruit may be detected on physical examination. Upper gastrointestinal hemorrhage as seen in around $2 / 3^{\text {rd }}$ of patients was the presenting complaint in our case [3].

Post-LC visceral artery pseudo-aneurysms have been reported as presenting anywhere between 5 days to 13 months [2,3]. Our case presented in 4 weeks. Depending on the time of presentation, bleeding may be minimal, or massive if presenting late $[2,3]$. The diagnosis of pseudo-aneurysm is difficult unless the condition is suspected. Ultrasound may reveal a hypo- echoic, pulsatile mass within the liver, with bidirectional flow on Doppler [3]. Contrast enhanced CT may demonstrate hemorrhage or pseudoaneurysm formation, which may be missed on ERCP and angiogram because of the intermittent character of the bleeding episode. Diagnosis of the source of bleeding can be made by UGI scopy, CT angiography with three-dimensional (3D) reconstruction, or angiography [3]. Because of its high diagnostic accuracy and therapeutic potential, angiography is considered a useful modality in the diagnosis and management of hemobilia with the reported success rate of angiographic control of hemobilia by trans-arterial embolization being $80 \%$ to $100 \%$ [2,5]. Early surgical intervention following failed radiologic intervention for hemobilia is often suggested, especially if embolization fails, or if cholecystitis, gallstones, or resectable neoplasms are present [2]. Our patient was successfully treated with trans-arterial embolization method with no rebleeds at 9 years of follow up.

\section{Conclusion}

Cystic artery pseudo-aneurysm is a complication of laparoscopic cholecystectomy which can potentially lead to a fatal emergency due to rupture. Diagnosis requires a high index of suspicion given the poor specificity of symptoms. These lesions are amenable to endoscopic and angiographic management, with low related morbidity and favorable long-term results. Any patient presenting with gastrointestinal bleeding post-laparoscopic cholecystectomy of either immediate or longer duration post-procedure needs to undergo urgent investigation with upper GI endoscopy and abdominal CT followed by angiography. Selective hepatic artery angiography is not only diagnostic, but can be used to embolize the pseudo-aneurysm. Early surgical ligation may be required in emergency cases where embolization fails.

Contributors: SAR: manuscript writing, and literature review and patient management; SM, OR: critical inputs into the manuscript and patient management. SAR will act as a study 
guarantor. All authors approved the final version of this manuscript and are responsible for all aspects of the study.

Funding: None; Competing interests: None stated.

\section{References}

1. Duca S, Bãlã O, Al-Hajjar N, Lancu C, Puia IC, Munteanu D, et al. Laparoscopic cholecystectomy: incidents and complications. A retrospective analysis of 9542 consecutive laparoscopic operations. HPB (Oxford). 2003;5:152-158.

2. Petrou A, Brennan N, Soonawalla Z, Silva MA. Hemobilia due to cystic artery stump pseudo aneurysm following laparoscopic cholecystectomy: case presentation and literature review. Int Surg. 2012;97:140144.

3. Roche-Nagle G, Maceneaney, Harte P. Pseudo-aneurysm of the hepatic artery after laparoscopic cholecystectomy: A case report. J Minim Access Surg. 2006;2:73-75.

4. Bin Traiki TA, Madkhali AA, Hassanain MM. Hemobilia post laparoscopic cholecystectomy. J Surg Case Rep. 20158;2015(2).

5. Maeda A, Kunou T, Saeki S, Aono K, Murata T, Niinomi N, et al. Pseudoaneurysm of the cystic artery with hemobilia treated by arterial embolization and elective cholecystectomy. J Hepatobiliary Pancreat Surg. 2002;9:755-758. 